\title{
Analysis and interpretation of the Seidel aberration coefficients in digital holography
}

\author{
Daniel Claus, ${ }^{1, \star}$ John Watson, ${ }^{2}$ and John Rodenburg ${ }^{1}$ \\ ${ }^{1}$ Kroto Research Institute, University of Sheffield, S3 7HQ, Sheffield, UK \\ ${ }^{2}$ Fraser Noble Building, University of Aberdeen, AB24 3UE, Aberdeen, UK \\ *Corresponding author: d.claus@sheffield.ac.uk
}

Received 1 August 2011; revised 3 November 2011; accepted 3 November 2011; posted 3 November 2011 (Doc. ID 151897); published 2 December 2011

\begin{abstract}
The most commonly used configurations in digital holography-namely Fourier holograms, Fresnel holograms, and image-plane holograms - are analyzed with respect to Seidel's wave aberration theory. This analysis is performed by taking into account the phase terms involved in the recording and reconstruction processes. The combined phase term from both processes is compared with the Gaussian-reference sphere, from which the wave aberration terms can be obtained. In conjunction with the analysis, for each of the aberration terms, conditions can be set to eliminate them. Wave aberrations are plotted to show how strongly different setups are affected. (C) 2011 Optical Society of America

OCIS codes: $\quad 090.1995,090.1000,080.1005$.
\end{abstract}

\section{Introduction}

Digital holography has become established as a versatile coherent imaging technique for the recovery of amplitude and phase information from an object. Typical holographic setups used in digital holography are Fresnel holograms, Fourier holograms, and image-plane holograms. A Fresnel hologram is characterized by the interference of a plane reference wave with the object wave. A Fourier hologram is characterized by an equidistantly located reference wave point source and object with respect to the sensor position. An image-plane hologram is recorded when the reference wave interferes with an in-focus image of the object. The above holographic setups can be implemented in in-line and off-axis configurations. For an in-line hologram, the locations of the reference wave point source, object, and camera sensor are coaxial. An off-axis hologram is defined by a reference wave angularly separated from the optic axis. The reconstructed hologram for an in-line setup is characterized by the overlap of the DC-term (undiffracted light), the real image, and the virtual image.

0003-6935/11/34H220-10\$15.00/0

(C) 2011 Optical Society of America
In order to suppress the DC-term and twin-image in the reconstruction of an in-line setup, phase stepping is implemented in the experiment. However, this results in an additional experimental effort, since it is necessary to record at least three phase stepped holograms. Temporal phase stepping using a piezomounted mirror in the reference arm is commonly applied. This restricts the setup to quasi-static events. Another way to suppress the appearance of the DC-term and twin-image is by using an off-axis setup. In the off-axis setup, the various components of the reconstructed hologram, such as the DC-term and the real and virtual images, are separated in the Fourier domain. In this manner, the desired component can be isolated, e.g., the real image. In the off-axis configuration, a single recorded hologram reveals the complex object information. The numerical reconstruction of the single hologram can be speeded up significantly by running the algorithm on the graphics card. This enables the application of real-time measurement for the investigation of dynamic events, as discussed in [1]. One major disadvantage of the off-axis geometry is the introduction of aberrations such as coma, astigmatism, and field curvature brought about by the oblique angle of the reference wave. Therefore, this paper is focused on the analysis 
of wave aberrations in conjunction with different holographic setups in the off-axis configuration.

To the authors' knowledge, the intrinsic appearance of wave aberrations for digital holographic setups has not been well explored yet. Various papers have been published for classical holography on this topic. Third-order aberrations have been explored by Meier [2] and Champagne [3]. Meier [2] derived expressions for spherical aberration, coma, astigmatism, field curvature, and distortion. Champagne [3] instead did not take into account field curvature and distortion, since they would only result in a change of position and not in a degradation of sharpness. Vikram and Billet [4] applied Meier's aberration expressions to analyze Fresnel holograms. Latta [5] derived fifth-order aberration terms. Hobson and Watson [6] discussed aberrations and their compensation introduced in submersible holography due to the change of the refractive index between the recording and reconstruction processes.

The analysis in this paper is based on the work of Meier [2], where the aberrations pertaining to optical (classical) holography have been discussed and quantified. In this paper Meier's work has been extended to cover a comparison of the most commonly setups in digital holography, such as Fourier, Fresnel, and image-plane holograms. A complete description of the aberration terms arising from different holographic configurations has been derived. In this manner, a two-dimensional complex conjugate phase map can be calculated, which enables the suppression of all aberration terms. The suppression of aberration terms in digital holography has been successfully demonstrated in various publications [7-11]. All these approaches require additional effort. One method requires the recording of an additional hologram without the object (see [7, 8]). Based on the intrinsic aberration immunity of double exposure holography, the two reconstructed holograms, with and without object, are then subtracted from each other. Other approaches rely on additional numerical effort by employing iterative curve fitting algorithms combined with Zernike polynomials (see [9-11]). An area close to the object is chosen as a reference area which should possess a constant phase in the situation where all aberrations and eventually arising curvature from the usage of a lens-system are suppressed. The fitting method does not require a priori knowledge about the setup configuration. However, it is based on the assumption of a flat and thin specimen.

In our case, successful aberration suppression or compensation depends on the exact measurement or determination of the reference source coordinates with respect to the recording wavelength. The various holographic configurations might respond differently to small measurement inaccuracies. Therefore this paper does not concentrate on aberration compensation but on the analysis of different holographic configurations with respect to the influence of small deviations from the reference source point coordinate.
The outcome of this study is beneficial in choosing the setup that is least sensitive to aberrations.

\section{Derivation of Aberrations in Digital Holography}

The aberration function $W\left(x^{\prime}, y^{\prime}\right)$ is defined by the Gaussian-reference sphere that passes through the center of the exit pupil. The exit pupil lies in the hologram-plane assuming that there is no physical aperture stop other than the hologram itself. The function $W\left(x^{\prime}, y^{\prime}\right)$ is positive or negative depending on whether the light rays travel a longer or shorter optical path length compared to the chief ray in reaching the Gaussian-reference sphere as shown in Fig. 1. In order to obtain the phase of the hologram, the phase terms involved in the recording and reconstruction processes are considered. In the recording process, an interference pattern resulting from the superposition of reference wave $\left(A_{\text {ref }} \exp \left(i \varphi_{\text {ref }}\right)\right)$ with the object wave $\left(A_{\text {obj }} \exp \left(i \varphi_{\text {obj }}\right)\right)$ is digitally stored in the hologram-plane. The recorded intensity pattern $\left(I\left(x^{\prime}, y^{\prime}\right)\right)$ can be mathematically described as

$$
\begin{aligned}
I\left(x^{\prime}, y^{\prime}\right)= & \left|A_{o b j} \exp \left(i \varphi_{o b j}\right)+A_{r e f} \exp \left(i \varphi_{r e f}\right)\right|^{2} \\
= & A_{o b j}^{2}+A_{r e f}^{2}+A_{o b j} A_{r e f} \exp \left[i\left(-\varphi_{o b j}+\varphi_{r e f}\right)\right] \\
& +A_{o} A_{r} \exp \left[i\left(\varphi_{o b j}-\varphi_{r e f}\right)\right],
\end{aligned}
$$

where $x^{\prime}$ and $y^{\prime}$ are the coordinates in the hologramplane (see Fig. 1). In the optical case, reconstruction is accomplished by illuminating the wet chemically processed hologram with a replica of the original reference wave. In digital holography, the wet chemical process is replaced by a multiplication of the digital hologram with the complex conjugated digital reference wave followed by numerical propagation to the reconstruction plane, as outlined in [12]. The reference wave can be obtained numerically or it can be recorded; see [8]. However, it needs to be pointed out that for complete aberration suppression, although the hologram has been recorded within the paraxial region in accordance with Eq. (2) (adapted from [13])

$$
d=\sqrt[3]{\frac{\pi}{4 \lambda}\left[\left(x^{\prime}-x\right)^{2}+\left(y^{\prime}-y\right)^{2}\right]^{2}}
$$

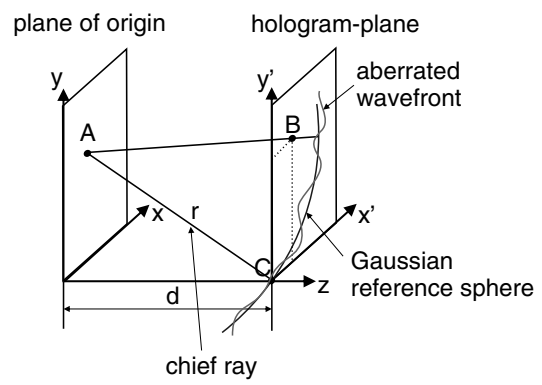

Fig. 1. Gaussian-reference sphere originating at $A$ and hologramplane intersection points $B$ and $C$. 
the complex conjugate reference wave cannot be approximated parabolically. This is due to the fact that the combination of the three phase terms results in a nonparabolic wavefront curvature. Significant phase-aberrations larger than 1 radian departure from the Gaussian-reference sphere are still obtained within the paraxial region. The validity of this argument will be proven at a later stage of this paper.

Since wave aberrations are defined with respect to the exit pupil (hologram-plane), the additional phase term caused by the numerical propagation to the reconstruction plane is not taken into account. Without loss of generality, only the phase of the real image is considered for further investigation. The phase of the real image is defined as

$$
\varphi_{\text {real }}=\varphi_{\text {rec }}-\varphi_{o b j}+\varphi_{r e f},
$$

where $\varphi_{\text {rec }}$ is the phase of the reconstruction wave. Now we need to find a formulation in which the phase terms are represented by the $x, y$, and $z$ coordinates of the object point and the point sources of reference and reconstruction waves. The wave aberrations are defined with respect to the Gaussianreference sphere, which passes through the center of the hologram $\left(x^{\prime}=0, y^{\prime}=0\right)$. Therefore the phase of the propagated wave needs to be compared with the spherical wave, originating from the same object point $A$ (see Fig. 1), but passing through the center of the hologram $C$.

$$
\varphi_{o b j}=k(\overline{A B}-\overline{A C}) .
$$

In order to describe the propagation from the object-plane to the hologram-plane with respect to the coordinates in both planes, the RayleighSommerfeld diffraction integral is used, as discussed in [13]. Moreover, the reference, object, and reconstruction waves are considered to be point sources in their corresponding planes of origin. Hence the resulting phase term for the object wave becomes

$$
\begin{aligned}
\varphi_{o b j}= & k d_{o b j}\left[\sqrt{1+\frac{\left(x^{\prime}-x_{o b j}\right)^{2}}{d_{o b j}^{2}}+\frac{\left(y^{\prime}-y_{o b j}\right)^{2}}{d_{o b j}^{2}}}\right. \\
& -\sqrt{\left.1+\frac{\left(x_{o b j}^{2}+y_{o b j}^{2}\right)}{d_{o b j}^{2}}\right]}
\end{aligned}
$$

with $k=\frac{2 \pi}{\lambda}$ the wave-number. A binomial expansion series is applied to the root expression:

$$
\sqrt{1+a}=1+\frac{1}{2} a-\frac{1}{8} a^{2}+\frac{1}{16} a^{3}-\cdots .
$$

The first two terms in Eq. (6) define the region of paraxial validity, which is then used to calculate the coordinates of the Gaussian-reference sphere. The third term is applied to calculate the third-order Seidel aberrations. Higher order terms than those are assumed to be of minor impact and are therefore neglected. Replacing the variable $a$ in Eq. (6) with the appropriate expression in Eq. (5) results in

$$
\begin{aligned}
\varphi_{o b j}= & k\left[\frac{1}{2 d_{o b j}}\left(x^{\prime 2}-2 x^{\prime} x_{o b j}-2 y^{\prime} y_{o b j}+y^{\prime 2}\right)\right. \\
& -\frac{1}{8 d_{o b j}^{3}}\left(x^{\prime 4}+y^{\prime 4}+2 x^{\prime 2} y^{\prime 2}-4 x^{\prime 3} x_{o b j}-4 y^{\prime 3} y_{o b j}\right. \\
& -4 x^{\prime 2} y^{\prime} y_{o b j}-4 y^{\prime 2} x^{\prime} x_{o b j}+6 x^{\prime 2} x_{o b j}^{2}+6 y^{\prime 2} y_{o b j}^{2} \\
& +8 x^{\prime} x_{o b j} y^{\prime} y_{o b j}+2 x^{\prime 2} y_{o b j}^{2}+2 y^{\prime 2} x_{o b j}^{2}-4 x^{\prime} x_{o b j}^{3} \\
& \left.\left.-4 y^{\prime} y_{o b j}^{3}-4 x^{\prime} x_{o b j} y_{o b j}^{2}-4 y^{\prime} y_{o b j} x_{o b j}^{2}\right)\right] .
\end{aligned}
$$

The corresponding phase terms $\varphi_{\text {ref }}$ and $\varphi_{\text {rec }}$ are derived for reference and reconstruction waves by replacing the subscript $o b j$ with $r e f$ or $r e c$, respectively. The Gaussian-reference sphere coordinates are obtained by the combination of the paraxial phase terms of $\varphi_{o b j}, \varphi_{r e f}$, and $\varphi_{r e c}$ according to Eq. (3). The Gaussian-reference sphere coordinates are indicated by the subscript $G$.

$$
\begin{aligned}
\frac{x^{\prime 2}-2 x^{\prime} x_{G}-2 y^{\prime} y_{G}+y^{\prime 2}}{d_{G}}= & \frac{\left(x^{\prime 2}-2 x^{\prime} x_{r e c}-2 y^{\prime} y_{r e c}+y^{\prime 2}\right)}{d_{r e c}} \\
& -\frac{\left(x^{\prime 2}-2 x^{\prime} x_{o b j}-2 y^{\prime} y_{o b j}+y^{\prime 2}\right)}{d_{o b j}} \\
& +\frac{\left(x^{\prime 2}-2 x^{\prime} x_{r e f}-2 y^{\prime} y_{r e f}+y^{\prime 2}\right)}{d_{r e f}} .
\end{aligned}
$$

The point source coordinates of the Gaussianreference sphere are then obtained in the following manner:

- For distance $d_{G}, \quad x_{G}=x_{r e c}=x_{o b j}=x_{r e f}=0$ and $y_{G}=y_{r e c}=y_{o b j}=y_{r e f}=0$.

- For $x_{G}, y_{G}=y_{r e c}=y_{o b j}=y_{r e f}=0$ and $y^{\prime}=0$.

- And for $y_{G}, x_{G}=x_{r e c}=x_{o b j}=x_{r e f}=0$ and $x^{\prime}=0$.

These equations result in the following expression for the point source coordinates of the Gaussianreference sphere:

$$
\begin{gathered}
\frac{1}{d_{G}}=\frac{1}{d_{r e c}}-\frac{1}{d_{o b j}}+\frac{1}{d_{r e f}}, \\
x_{G}=\left(\frac{x_{r e c}}{d_{r e c}}-\frac{x_{o b j}}{d_{o b j}}+\frac{x_{r e f}}{d_{r e f}}\right) d_{G}, \\
y_{G}=\left(\frac{y_{r e c}}{d_{r e c}}-\frac{y_{o b j}}{d_{o b j}}+\frac{y_{r e f}}{d_{r e f}}\right) d_{G} .
\end{gathered}
$$

The two most commonly applied methods for the description of wave aberrations are Zernike polynomials and Seidel coefficients. Zernike polynomials 
are often used in interferometry to isolate and suppress possible arising aberration terms, because they are unconstrained by symmetry, as discussed in [14]. Seidel coefficients, on the other hand, are derived from an expansion series. A strict correlation between both Zernike polynomials and Seidel coefficients only exists where orders higher than third are negligible. Higher-order Zernike polynomials can contain lower-order Seidel coefficients; see [14]. Seidel sum coefficients have been used to describe the aberration terms represented by the combination of the third-order terms in Eq. (7). This choice is due to the fact that both Seidel sum coefficients and the third-order terms in Eq. (7) show a strong similarity in the mathematical description (expansion series). This eases the extraction of Seidel sum coefficients from Eq. (7). Moreover, each aberration coefficient for Seidel sum coefficients is obtained from a summation over all surfaces, or in our case, all different contributing waves (reference wave, object wave, reconstruction wave). Seidel sum coefficients for different wave aberration terms are as follows:

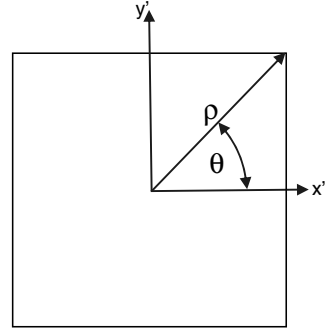

Fig. 2. Radial pupil coordinates $\rho$ and $\theta$.

aberrations involved. We first of all consider lensless setups and compare Fresnel and Fourier holograms.

\section{A. Fresnel Hologram}

A Fresnel hologram is characterized by having a plane reference wave and plane reconstruction wave, which results in an infinity reference point source distance $d_{r e f}$ and reconstruction point source distance $d_{\text {rec }}$; see Fig. 3 .

$$
d_{\text {ref }}=d_{\text {rec }}= \pm \infty \text {. }
$$

$$
\begin{aligned}
W(\rho, \theta)= & \frac{2 \pi}{\lambda} \cdot\left[\frac{1}{8} \rho^{4} S_{I}\right. & & \text { Spherical aberration } \\
& +\frac{1}{2} \rho^{3}\left(S_{I I y} \cos \theta+S_{I I x} \sin \theta\right) & & \text { Coma } \\
& +\frac{1}{2} \rho^{2}\left(S_{I I I x} \cos ^{2} \theta+S_{I I I y} \sin ^{2} \theta+2 S_{I I I x y} \cos \theta \sin \theta\right) & & \text { Astigmatism } \\
& +\frac{1}{4} \rho^{2}\left(S_{I I I}+S_{I V}\right) & & \text { Field curvature } \\
& \left.+\frac{1}{2} \rho\left(S_{V x} \cos \theta+S_{V y} \sin \theta\right)\right] & & \text { Distortion, }
\end{aligned}
$$

where $\rho$ is the radius in the hologram-plane $\left(\rho=\sqrt{x^{\prime 2}+y^{\prime 2}}\right)$ and $\theta$ is the angle $\left(\theta=\operatorname{acos}\left(\frac{x^{\prime}}{\rho}\right)=\right.$ $\left.\operatorname{asin}\left(\frac{y^{\prime}}{\rho}\right)\right)$; see Fig. 2. By comparison of the third-order terms in Eq. (6) with the aberration parameters described in Eq. (12), Seidel's sum coefficients are obtained.

The one-dimensional Seidel sum coefficients for astigmatism $S_{I I I x}$ and $S_{I I I y}$, shown in Table 1 , differ from those calculated by Meier [2] by a factor of 1.5 , although the same set of equations was used. The additional factor for the astigmatic aberration coefficient arises from the comparison of the two phase terms presented in Eq. (7) $\left(6 x^{\prime 2} x_{\mathrm{obj}}^{2}\right.$ and $6 y^{\prime 2} y_{\mathrm{obj}}^{2}$ ) with the corresponding Seīdel's sum coefficients represented in Eq. (12). To prove this fact, the derivation of $S_{\text {IIIx }}$ with respect to the object coordinates is shown in the following:

\begin{tabular}{|c|c|}
\hline $\begin{array}{l}\text { Aberration } \\
\text { term }\end{array}$ & Seidel's sum coefficient \\
\hline $\begin{array}{l}\text { Spherical } \\
\text { aberration }\end{array}$ & $S_{I}=-\left(\frac{1}{d_{r e c}^{3}}-\frac{1}{d_{o b j}^{3}}+\frac{1}{d_{r e f}^{3}}-\frac{1}{d_{G}^{3}}\right)$ \\
\hline Coma & $S_{I I x}=+\left(\frac{x_{r e c}}{d_{r e c}^{3}}-\frac{x_{o b j}}{d_{o b j}^{3}}+\frac{x_{r e f}}{d_{r e f}^{3}}-\frac{x_{G}}{d_{G}^{3}}\right)$ \\
\hline Coma & $S_{I I y}=+\left(\frac{y_{r e c}}{d_{r e c}^{3}}-\frac{y_{o b j}}{d_{o b j}^{3}}+\frac{y_{r e f}}{d_{r e f}^{3}}-\frac{y_{G}}{d_{G}^{3}}\right)$ \\
\hline Astigmatism & $S_{I I I x}=-\frac{3}{2}\left(\frac{x_{r e c}^{2}}{d_{r e c}^{3}}-\frac{x_{o b j}^{2}}{d_{o b j}^{3}}+\frac{x_{r e f}^{2}}{d_{r e f}^{3}}-\frac{x_{G}^{2}}{d_{G}^{3}}\right)$ \\
\hline Astigmatism & $S_{I I I y}=-\frac{3}{2}\left(\frac{y_{r e c}^{2}}{d_{r e c}^{3}}-\frac{y_{o b j}^{2}}{d_{o b j}^{3}}+\frac{y_{r e f}^{2}}{d_{r e f}^{3}}-\frac{y_{G}^{2}}{d_{G}^{3}}\right)$ \\
\hline Astigmatism & $S_{I I I x y}=-\left(\frac{x_{r e c} y_{r e c}}{d_{r e c}^{3}}-\frac{x_{o b j} y_{o b j}}{d_{o b j}^{3}}+\frac{x_{r e f} y_{r e f}}{d_{r e f}^{3}}-\frac{x_{G} y_{G}}{d_{G}^{3}}\right)$ \\
\hline Field curvature & e $\quad S_{I V}=-\left(\frac{x_{r e c}^{2}+y_{r e c}^{2}}{d_{r e c}^{3}}-\frac{x_{o b j}^{2}+y_{o b j}^{2}}{d_{o b j}^{3}}+\frac{x_{r e f}^{2}+y_{r e f}^{2}}{d_{r e f}^{3}}-\frac{x_{G}^{2}+y_{G}^{2}}{d_{G}^{3}}\right)$ \\
\hline Distortion & $S_{V x}=+\left(\frac{x_{r e c}^{3}+x_{r e} y_{r e c}^{2}}{d_{r e c}^{3}}-\frac{x_{o b j}^{3}+x_{o b j} y_{o b j}^{2}}{d_{o b j}^{3}}+\frac{x_{r e f}^{3}+x_{r e f} y_{r e f}^{2}}{d_{r e f}^{3}}-\frac{x_{G}^{3}+x_{G} y_{G}^{2}}{d_{G}^{3}}\right)$ \\
\hline Distortion & $S_{V y}=+\left(\frac{y_{r e c}^{3}+y_{r e c} x_{r e c}^{2}}{d_{r e c}^{3}}-\frac{y_{o b j}^{3}+y_{o b j} x_{o b j}^{2}}{d_{o b j}^{3}}+\frac{y_{r e f}^{3}+y_{r e f} x_{r e f}^{2}}{d_{r e f}^{3}}-\frac{y_{G}^{3}+y_{G} x_{G}^{2}}{d_{G}^{3}}\right)$ \\
\hline
\end{tabular}

$$
\begin{aligned}
-\frac{2 \pi}{8 d_{o b j}^{3} \lambda}\left(6 x^{\prime 2} x_{o b j}^{2}\right) & =\frac{2 \pi}{\lambda} \frac{1}{2} \rho^{2} S_{I I I x} \cos ^{2} \theta \Rightarrow S_{I I I x} \\
& =-\frac{3}{2} \frac{x_{o b j}^{2}}{d_{o b j}^{3}}
\end{aligned}
$$

We now apply Seidel's sum coefficients to different holographic configurations in order to obtain the
Equation (14) is then applied to Eqs. (9), (10), and (11) in order to derive the corresponding Seidel coefficients for a Fresnel hologram. It needs to be pointed out that terms $\frac{x_{i}}{d_{i}}$ and $\frac{y_{i}}{d_{i}}$ (with $i=r e f, r e c$ ),

Table 1. Seidel Aberrations 


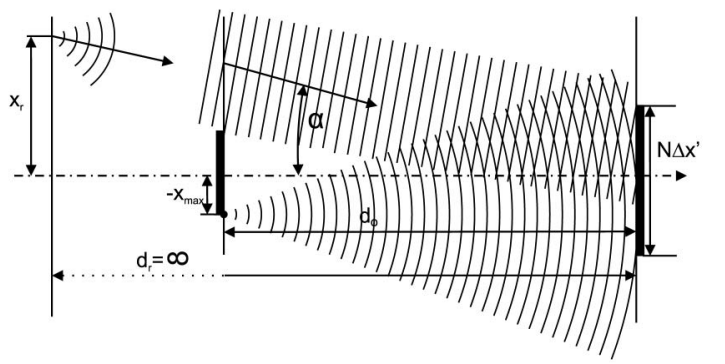

Fig. 3. Geometric configuration for a Fresnel hologram.

although of small magnitude, remain finite. This term is physically represented in the setup by the tangents of the off-axis angle $\alpha$ between the object and the corresponding reference or reconstruction wave, as shown in Fig. 3.

$$
\begin{gathered}
\frac{1}{d_{G}}=-\frac{1}{d_{o b j}}, \\
x_{G}=\left(-\frac{x_{r e c}}{d_{r e c}}+\frac{x_{o b j}}{d_{o b j}}-\frac{x_{r e f}}{d_{r e f}}\right) d_{o b j}, \\
y_{G}=\left(-\frac{y_{r e c}}{d_{r e c}}+\frac{y_{o b j}}{d_{o b j}}-\frac{y_{r e f}}{d_{r e f}}\right) d_{o b j} .
\end{gathered}
$$

The resulting Seidel sum coefficients are calculated and if an aberration term is not eliminated by setting an appropriate initial distance (Eq. (14)), conditions are given in order to nullify the corresponding aberration term. Both Seidel's sum coefficients and the corresponding conditions are shown in Table 2.

\section{B. Fourier Hologram}

The same procedure is applied to Fourier holograms. A Fourier hologram is characterized by having an equidistantly positioned reference point source $x_{\text {ref }}$ and object point source $x_{o b j}$ with respect to the camera position, as shown in Fig. 4 . The reconstruction of the real image for a Fourier hologram is obtained by utilizing a convergent reconstruction wave. We can therefore write

$$
d_{r e f}=d_{o b j}=-d_{r e c} .
$$

This results in the following coordinates for the Gaussian-reference sphere:

$$
\begin{gathered}
\frac{1}{d_{G}}=\frac{1}{d_{r e c}}=-\frac{1}{d_{o b j}}, \\
x_{G}=x_{r e c}+x_{o b j}-x_{r e f}, \\
y_{G}=y_{r e c}+y_{o b j}-y_{r e f} .
\end{gathered}
$$

Seidel's sum aberration coefficients are obtained by inserting Eqs. (19), (20), and (21) into the equations shown in Table 1 , the results of which are shown in Table 3.

The phase aberration terms for corresponding Seidel aberration coefficients for an increasing ratio $\left(\frac{x_{\text {ref }}}{d_{\text {ref }}}\right)$ have been calculated for the Fresnel and Fourier cases with a recording wavelength of $0.5 \mu \mathrm{m}$, a $1024 \times$ 1024 pixel sensor with a pixel-size of $5 \mu \mathrm{m}$, and a $60 \mathrm{~mm}$ object-size $\left(2 \cdot x_{\max }\right.$; see Figs. 3 and 4 for an explanation of parameters used). The object distance $d_{o b j}$ used was chosen to match the minimum recording distance at which the paraxial approximation becomes valid; see Eq. (22). In this manner, it could be proven that significant third-order aberrations arise

\begin{tabular}{|c|c|c|}
\hline Aberration term & Seidel's sum coefficient & Conditions \\
\hline Spherical aberration & 0 & \\
\hline Coma & $S_{I I x}=-\frac{1}{d_{o b i}^{2}}\left(\frac{x_{r e c}}{d_{r e c}}+\frac{x_{r e f}}{d_{r e f}}\right)$ & $x_{\text {rec }}=-x_{\text {ref }}$ \\
\hline Coma & $S_{I I y}=-\frac{1}{d_{o b j}^{2}}\left(\frac{y_{r e c}}{d_{r e c}}+\frac{y_{r e f}}{d_{r e f}}\right)$ & $y_{r e c}=-y_{\text {ref }}$ \\
\hline Astigmatism & 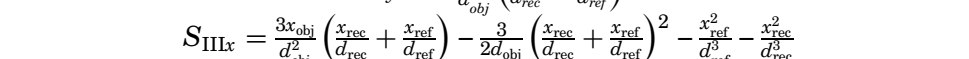 & $\frac{x_{\mathrm{rec}}}{d_{\mathrm{rec}}}=-\frac{x_{\mathrm{ref}}}{d_{\mathrm{ref}}}$ \\
\hline Astigmatism & 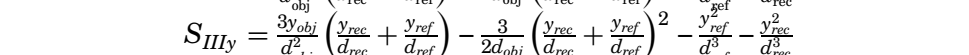 & $\frac{y_{\mathrm{rec}}}{d_{\mathrm{rec}}}=-\frac{y_{\mathrm{ref}}}{d_{\mathrm{ref}}}$ \\
\hline Astigmatism & 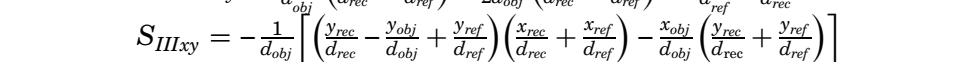 & 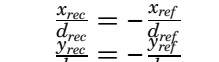 \\
\hline Field curvature & $S_{I V}=-\frac{1}{d_{o b j}}\left[-\frac{2 x_{o b j}}{d_{o b j}}\left(\frac{x_{r e c}}{d_{r e c}}+\frac{x_{r e f}}{d_{r e f}}\right)-\frac{2 y_{o b j}}{d_{o b j}}\left(\frac{y_{r e c}}{d_{r e c}}+\frac{y_{r e f}}{d_{r e f}}\right)+\left(\frac{x_{r e c}}{d_{r e c}}+\frac{x_{r e f}}{d_{r e f}}\right)^{2}+\left(\frac{y_{r e c}}{d_{r e c}}+\frac{y_{r e f}}{d_{r e f}}\right)^{2}\right]$ & 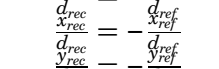 \\
\hline Distortion & 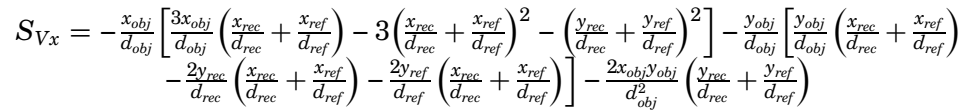 & $\begin{array}{l}\frac{d_{\text {rec }}}{x_{\text {rec }}}=-\frac{d_{\text {ree }}}{d_{\text {rec }}} \\
\frac{y_{\text {rec }}}{d_{\text {ree }}}=-\frac{y_{\text {ref }}}{d_{\text {ref }}}\end{array}$ \\
\hline Distortion & $\begin{array}{l}-\frac{x_{r e f}}{d_{r e f}}\left(\frac{3 x_{r e c}^{2}}{d_{r e c}^{2}}+\frac{2 y_{r e f} y_{r e c}}{d_{r e f}}+\frac{y_{r e c}^{2}}{d_{r e c}^{2}}\right)-\frac{x_{r e c}}{d_{r e c}}\left(\frac{3 x_{r e f}^{2}}{d_{r e f}^{2}}+\frac{2 y_{r e f} y_{r e c}}{d_{r e f}}+\frac{y_{r e c}^{2}}{d_{r e f}^{2}}\right) \\
S_{V y} \text { is found be replacing } x \text { with } y \text { and vice versa }\end{array}$ & same as for $S_{V x}$ \\
\hline
\end{tabular}
within the paraxial region.

$$
d_{o b j}=\sqrt[3]{\left[\left(x^{\prime}-x\right)^{2}+\left(y^{\prime}-y\right)^{2}\right]^{2} \frac{\pi}{4 \lambda}} .
$$

For the parameters above, the minimum recording distance becomes $1920 \mathrm{~mm}$.

Table 2. Fresnel Hologram Seidel Aberrations 


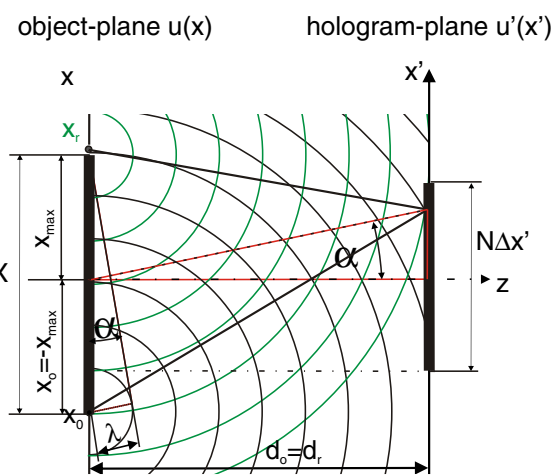

Fig. 4. (Color online) Geometric configuration of a Fourier hologram.

Without loss of generality, only the $x$-components of each of the corresponding aberration terms have been taken into account. To simplify the calculation (without loss of generality in further conclusions), rather than considering all object points, only the object point that contributes to the largest magnitude of aberration was taken into account. This object point is positioned furthest from the reference source point as shown in Figs. 3 and 4. Moreover, the tangents of the reconstruction angle $\alpha^{\prime}$ were set to zero $\left(\tan \alpha^{\prime}=\frac{x_{\text {rec }}}{d_{\text {rec }}}=0\right)$ ). The results of this investigation are graphically represented in Fig. 5 .

Aberration terms were also plotted for increasing and decreasing pixel-size $\Delta x^{\prime}$, pixel-number $N$, wavelength $\lambda$, and object-size $X=2 x_{\max }$, while only the corresponding parameter was changed and the others kept at its initial value. Rather than displaying those plots, the trend of the corresponding aberration term for the increasing parameter is indicated in the graphs of Fig. 5 by a black arrow. The graphs for astigmatism, field curvature, and the sum of all third-order aberrations can be split into two sections. The first section is defined by those values of $\frac{x_{r e f}}{d_{r e f}}$ that are smaller than the point at which the aberration curve intersects the zero-line (black dot-dashed line). Whereas the second section is defined for values larger than the point of intersection. In the first section, the wavelength has a strong impact on the shape of the curve and the position of the intersection point. For increasing wavelength, the curvature increases and the point of intersection is shifted toward larger $\frac{x_{\text {ref }}}{d_{\text {ref }}}$ values. For the sum of aberrations, the wavelength has a stronger impact on the curve and position of the intersection point when using a Fourier hologram rather than a Fresnel hologram. Another parameter that strongly influences the shape of the aberration curves is the pixel-size. Reducing the pixel-size is comparable with cropping a section of the graph and extending this section to cover whole the range on the abscissa. This effect can be explained by the reduction of the sensor area, when reducing the pixel-size only.

From all these curves, it can be generalized that in close proximity of the coordinates of the real reference wave (roughly $10 \mathrm{mrad}$ ), a Fourier hologram is less strongly affected by aberrations than a Fresnel hologram.

\section{Image-Plane Hologram}

An image-plane hologram is characterized by a lens or lens-system that projects an image of the object onto the camera sensor; see Fig. 6 . In this manner, the reconstruction of the hologram is not required. The phase of the object wave for an image-plane hologram, based on the assumption of an aberrationfree and laterally infinitely extent lens-system, can be described as (adapted from [13])

$$
\varphi_{o b j}=k\left(\frac{M+1}{M}\right)\left[\frac{1}{2} \frac{x^{\prime 2}+y^{\prime 2}}{d_{2}}-\frac{1}{8} \frac{x^{\prime 4}+2 x^{\prime 2} y^{\prime 2}+y^{\prime 4}}{d_{2}^{3}}\right],
$$

where $M$ is the lens-system's magnification. Applying the paraxial approximation results in the coordinates of the Gaussian-reference sphere:

$$
\begin{aligned}
\varphi_{G} & =\varphi_{r e f}-\varphi_{o b j} \\
& =\frac{x^{\prime 2}-2 x^{\prime} x_{r e f}-2 y^{\prime} y_{r e f}+y^{\prime 2}}{d_{r e f}}-\frac{M+1}{M} \frac{x^{\prime 2}+y^{\prime 2}}{d_{2}} .
\end{aligned}
$$

\begin{tabular}{|c|c|c|}
\hline Aberration term & Seidel's sum coefficient & Conditions \\
\hline $\begin{array}{l}\text { Spherical } \\
\text { aberration }\end{array}$ & 0 & 0 \\
\hline Coma & 0 & 0 \\
\hline Coma & 0 & 0 \\
\hline Astigmatism & $S_{I I I x}=\frac{3}{d^{3}}\left(x_{o b j}-x_{r e f}\right)\left(x_{r e f}-x_{r e c}\right)$ & $x_{r e f}=x_{r e c}$ or $x_{r e f}=x_{o b j}$ \\
\hline Astigmatism & $S_{I I I y}=\frac{3}{d^{3}}\left(y_{o b j}-y_{r e f}\right)\left(y_{r e f}-y_{r e c}\right)$ & $y_{\text {ref }}=y_{\text {rec }}$ or $y_{\text {ref }}=y_{o b j}$ \\
\hline Astigmatism & $S_{I I I x y}=-\frac{1}{d_{o b j}^{3}}\left[\left(y_{r e c}-y_{r e f}\right)\left(x_{o b j}-x_{r e f}\right)+\left(y_{o b j}-y_{r e f}\right)\left(x_{r e c}-x_{r e f}\right)\right]$ & $\begin{array}{c}\left(y_{r e c}=y_{r e f} \text { or } x_{o b j}=x_{r e f}\right) \text { and }\left(y_{o b j}=y_{r e f} \text { or }\right. \\
\left.x_{r e c}=x_{r e f}\right)\end{array}$ \\
\hline Field curvature & $S_{I V}=-\frac{2}{d_{o b j}^{3}}\left[\left(x_{o b j}-x_{r e f}\right)\left(x_{r e c}-x_{r e f}\right)+\left(y_{o b j}-y_{r e f}\right)\left(y_{r e c}-y_{r e f}\right)\right]$ & $\begin{array}{c}\left(x_{o b j}=x_{r e f} \text { or } x_{r e c}=x_{r e f}\right) \text { and }\left(y_{o b j}=y_{r e f} \text { or }\right. \\
\left.y_{r e c}=y_{r e f}\right)\end{array}$ \\
\hline Distortion & $\begin{array}{c}S_{V x}=\frac{1}{d_{o b j}^{3}}\left[\left(x_{r e f}-x_{r e c}\right)\left\{3 x_{o b j}\left(x_{r e f}-x_{r e c}\right)-3 x_{o b j}^{2}-y_{o b j}^{2}+3 x_{r e f} x_{r e c}+2 y_{r e c} y_{r e f}\right\}\right. \\
+\left(y_{r e f}-y_{r e c}\right)\left\{x_{o b j}\left(y_{r e f}-y_{r e c}\right)\right. \\
\left.\left.-2 y_{o b j}\left(x_{r e c}+x_{o b j}-x_{r e f}\right)\right\}-x_{r e f} y_{r e c}^{2}+x_{r e c} y_{r e f}^{2}\right]\end{array}$ & $x_{r e c}=x_{r e f}$ and $y_{r e c}=y_{r e f}$ \\
\hline Distortion & $S_{V y}$ is found be replacing $x$ with $y$ and vice versa & same as for $S_{V x}$ \\
\hline
\end{tabular}

Hence the coordinates are

Table 3. Fourier Hologram Seidel Aberrations 


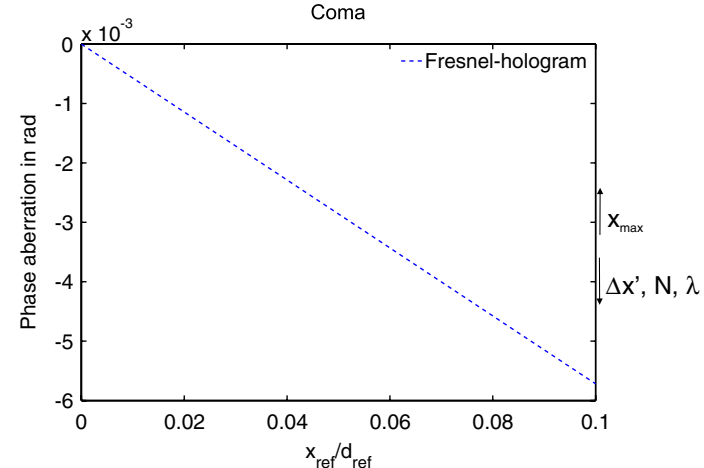

(a)

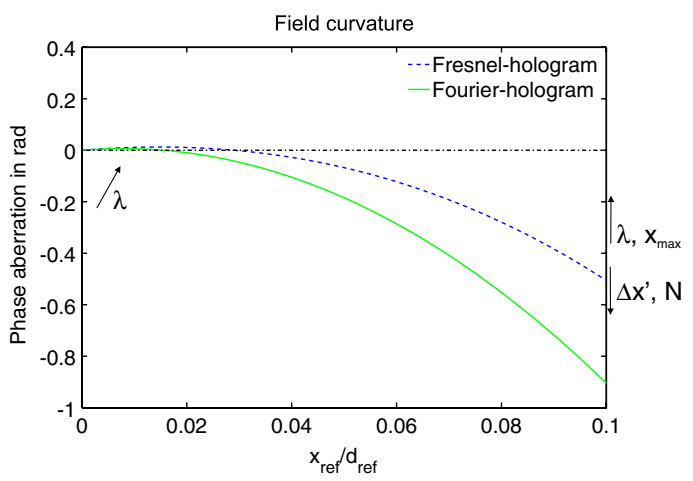

(c)

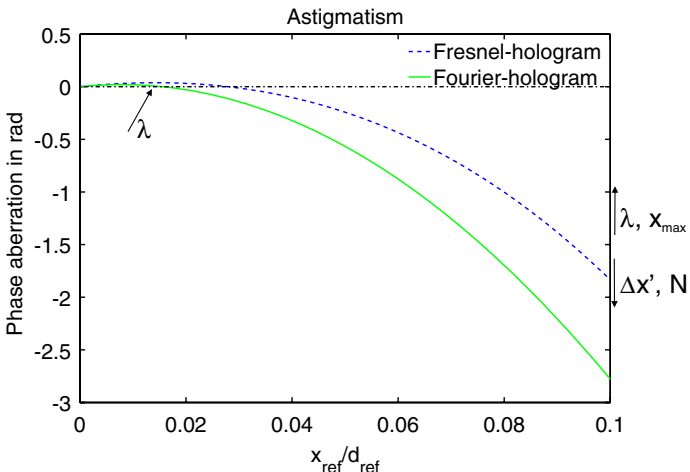

(b)

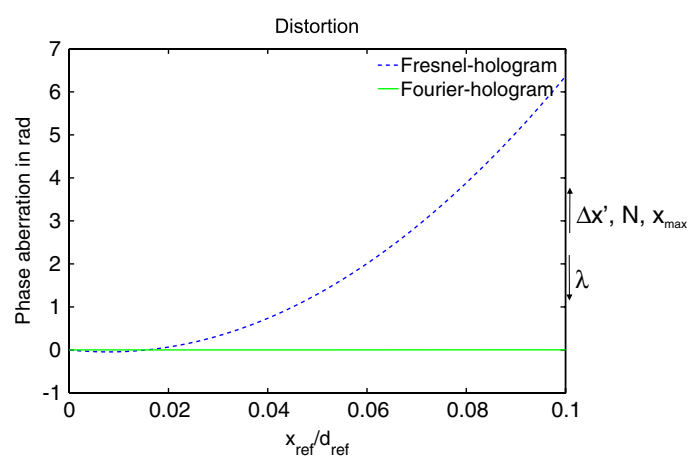

(d)

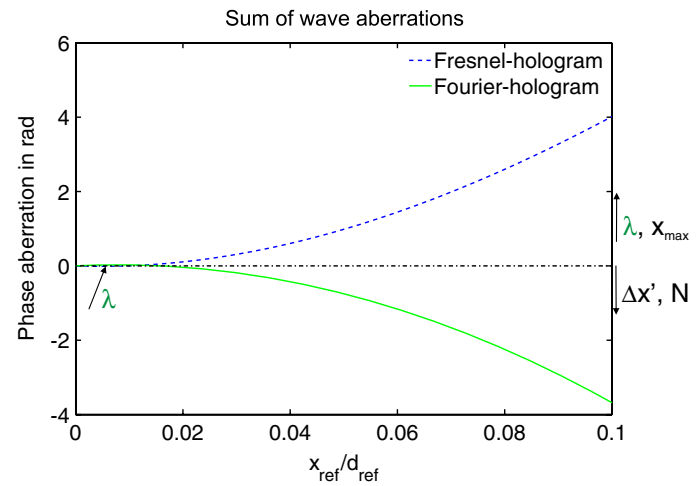

(e)

Fig. 5. (Color online) Phase-aberrations for Fresnel hologram and Fourier hologram: (a) Coma, (b) astigmatism, (c) field curvature, (d) distortion, and (e) combination of all terms.

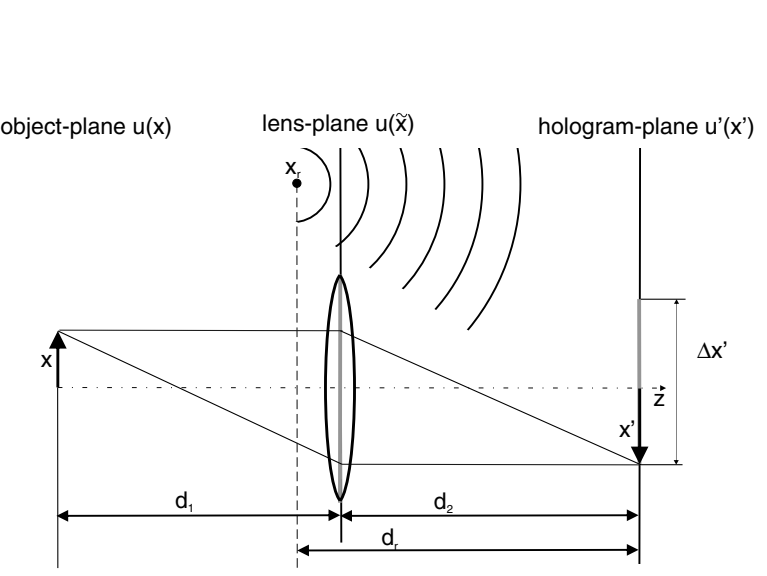

Fig. 6. Geometric configuration of an image-plane hologram.

$$
\frac{1}{d_{G}}=\frac{1}{d_{r e f}}-\frac{M+1}{M d_{2}}, x_{G}=d_{G} \frac{x_{r e f}}{d_{r e f}}, y_{G}=d_{G} \frac{y_{r e f}}{d_{r e f}} .
$$

\section{Table 4. Image-Plane Hologram Seidel Aberrations}

\begin{tabular}{|c|c|}
\hline Aberration term & Seidel's sum coefficient \\
\hline Spherical aberration & $S_{I}=-\left(\frac{1}{d^{3}}-\frac{M+1}{M} \frac{1}{d^{3}}-\frac{1}{d^{3}}\right)$ \\
\hline Coma & $S_{I I x}=\frac{x_{\text {ref }}}{d^{3}}-\frac{x_{G}^{2}}{d_{G}^{3}}$ \\
\hline Astigmatism & $S_{I I I x}=-\frac{3}{2}\left(\frac{x_{e f}^{2}}{d_{r e f}^{2}}-\frac{x_{G}^{2}}{d_{G}^{3}}\right)$ \\
\hline Astigmatism & $S_{I I I x y}=-\left(\frac{x_{r e f} y_{\text {ref }}}{d_{r e f}^{3}}-\frac{x_{G} y_{G} y_{G}}{d_{G}^{3}}\right)$ \\
\hline Field curvature & $S_{I V}=-\left(\frac{x_{r e f}^{2}+y_{r e f}^{2}}{d^{3}}-\frac{x_{G}^{2}+y_{G}^{2}}{d^{3}}\right)$ \\
\hline Distortion & $S_{V}=0$ \\
\hline
\end{tabular}




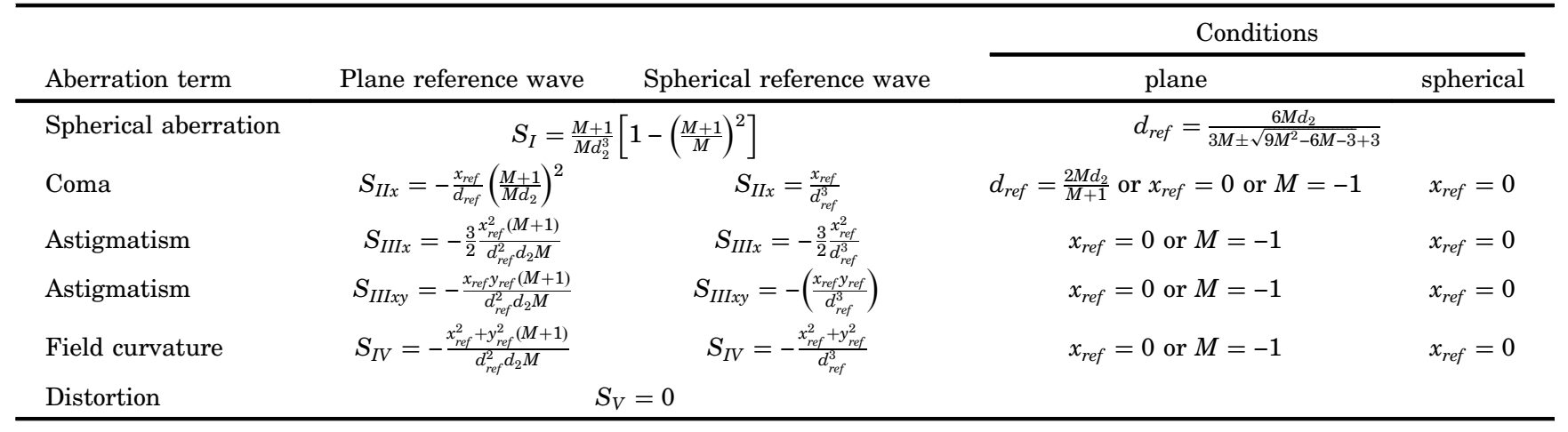

In the following, Seidel's sum coefficients for an image-plane hologram have been derived; see Table 4. Because of the similarity between Seidel's sum coefficients for $x$ and $y$ coordinates, only the $x$ coordinate and the mixed terms are considered hereafter.

Two different types of image-plane holograms have been analyzed with respect to the axial position of the reference wave point source. In the first case, the reference wave point source is positioned at infinity, resulting in plane wavefronts. In the second case, the distance of the spherical reference wave was chosen to obtain a matched reference wavefront and object wavefront $\left(d_{r e f}=\frac{d_{2} M}{M+1}\right)$.

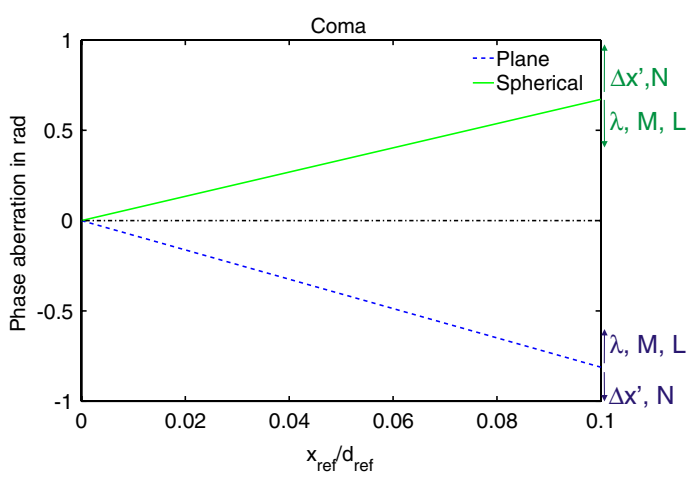

(a)

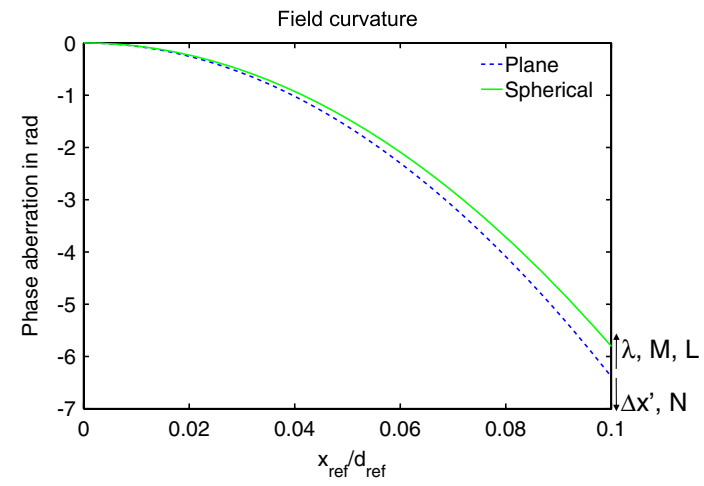

(c)
The results obtained are shown in Table 5. They have then been used to plot the phase aberration terms for the $x$-coordinate only. The image distance $d_{2}$ as shown in Fig. 6 can be obtained from the magnification and the object distance $d_{1}$.

$$
d_{2}=d_{1} M
$$

The distance between object and image is standardized to $195 \mathrm{~mm}$ for conventional noninfinity microscope objectives (see ISO 9345-1). This enables the calculation of $d_{2}$, although $d_{1}$ might be unknown.

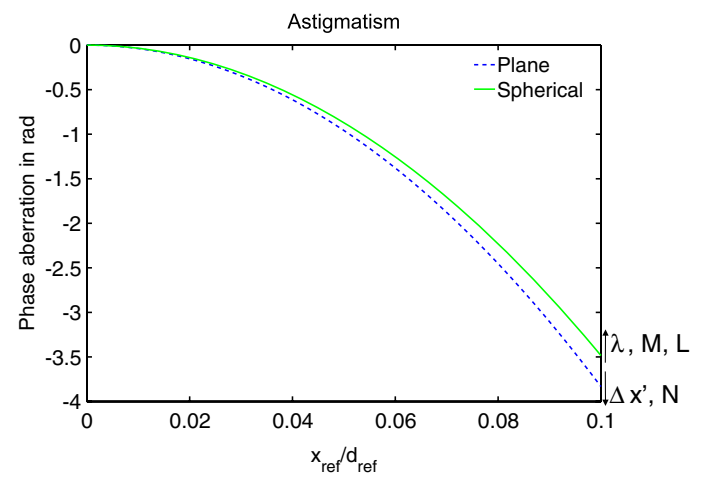

(b)

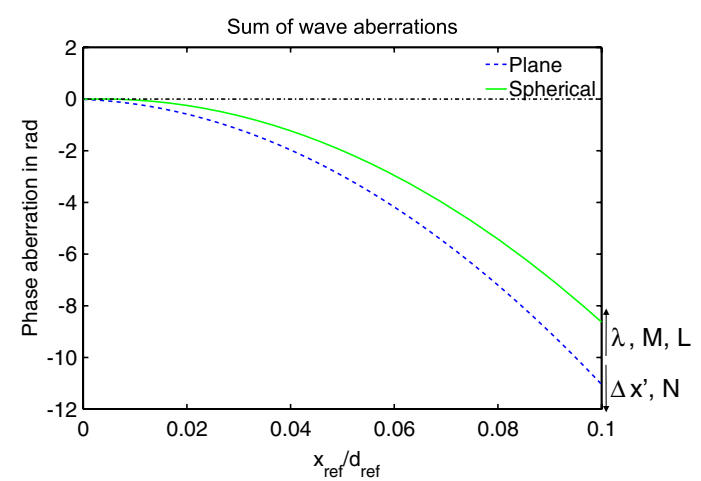

(d)

Fig. 7. (Color online) Phase-aberrations for image-plane hologram with plane and spherical reference wave: (a) Coma, (b) astigmatism, (c) field curvature, and (d) combination of all terms. 


$$
d_{2}=\frac{\left(d_{2}+d_{1}\right) M}{1+M}=\frac{L \cdot M}{1+M} .
$$

The plotted phase aberration terms for a $10 \times$ microscope objective $(M=10, L=195 \mathrm{~mm})$ and the same wavelength and sensor specifications as previously used are shown in Fig. 7. By analogy with the analysis of lensless holograms, the parameters have been alternated to obtain their influence on the shape of the corresponding aberration curve. Contrary to lensless holography, the object-size has not been taken into account, since an aberration free lens-system was assumed. However, the magnification $M$ and the distance from the object to the sensor $L$ were considered for this analysis. If the lens aberrations had to be accounted for, then they would contribute to the overall aberrations in an additive manner and hence would not change the conclusions drawn.

Over the entire range defined by the abscissa, representing the tangents of the reference wave angle, an object wave adapted spherical reference wave resulted in reduced aberrations in comparison with a plan reference wave. Wave aberrations increased with increasing pixel-size $\Delta x^{\prime}$ and pixel-number $N$. This was expected since it results in an increased sensor area. On the other hand, an increasing magnification $M$, object-sensor distance $L$, and wavelength $\lambda$ results in decreased aberration magnitudes.

\section{Discussion and Conclusion}

In the case of an off-axis hologram, it is important to measure the recording coordinates of the reference wave to as high a precision as possible with respect to the wavelength employed. In that manner, a good match of the numerical reference wave to the real one is generated, which results in a less aberrated reconstruction.

In situations where the reference wave coordinates cannot be determined with sufficient accuracy but are still within close proximity (the deviation between the reference wave angle determined and the real angle is smaller than $0.01 \mathrm{rad}$ ) to the true coordinates, the wave aberrations involved are smaller with a Fourier hologram rather than a Fresnel hologram; see Fig. 5.

A reduction in the impact of the aberrations could also be confirmed for image-plane holography with a spherical reference wave whose curvature matches the wavefronts, leaving the microscope objective. The difference between the aberration curve for plane and spherical reference wave increases when a lens or lens-system of smaller magnification is employed. This suggests that wave aberrations for a plane reference wave increase with decreasing magnification.

It is also very common in digital holographic microscopy to record an out-of-focus image in front of the image-plane, as shown in [15]. This setup results in an increased field-of-view by which the spacebandwidth product of the sensor is more efficiently used, as demonstrated in [16]. In this manner, empty magnification can be avoided without needing to pay for a highly expensive microscope objective of the same numerical aperture but smaller magnification. The term "empty magnification" indicates that more than the ideal number of one pixel is used in order to display an object detail. It is then necessary to adapt the curvature of the complex conjugate reference wave to the reference wave in the out-of-focus recording position.

Both the recording of a lensless hologram and an image-plane hologram support the idea of using a spherical reference wave in the recording process. Moreover, a spherical reference wave enables a goodquality wavefront without needing to insert any additional optical elements between the reference wave origin and camera. In this manner, the reference wave is not affected by lens aberrations, which can be introduced in the collimation process in order to generate a plane reference wave, which is needed for a Fresnel hologram. Furthermore, the lenssystem required for the collimation process of a Fresnel hologram can be affected by dust on the lens surfaces, which negatively influences the quality of the reference wave. Another benefit when employing Fourier holograms is that the reference point source can be positioned adjacent to the object, which results in an almost common-path interferogram, thereby increasing the environmental stability of the arrangement.

The findings of this investigation are beneficial in the selection of hologram geometry that will possess the least aberrations in any given situation. Moreover, Seidel sum coefficients derived can be used to calculate the magnitude of any aberrations present and their numerical suppression. The suppression of aberrations in digital holography could be demonstrated by [7] and [8], where Seidel's sum coefficients were not considere $\bar{d}$. Taking account of Seidel's coefficient in selecting the most appropriate recording geometry can result in a more direct and less timeconsuming solution in order to numerically suppress any wave aberration that may be introduced.

\section{References}

1. T. Haist, M. Reicherter, M. Wu, and L. Seifert, "Using graphics boards to compute holograms," Comput. Sci. Eng. 8, 8-13 (2006).

2. R. W. Meier, "Magnification and third-order aberrations in holography," J. Opt. Soc. Am. 55, 987-992 (1965).

3. E. B. Champagne, "Nonparaxial imaging, magnification, and aberration properties in holography," J. Opt. Soc. Am. A 57, 51-55 (1967).

4. C. S. Vikram, and M. L. Billet, "Aberration limited resolution in fraunhofer holography with collimated beams," Opt. Laser Technol. 21, 173-184 (1989).

5. J. N. Latta, "Fifth-order hologram aberrations," Appl. Opt. 10, 666-667 (1971)

6. P. R. Hobson, and J. Watson, "The principles and practice of holographic recording of plankton," J. Opt. A 4, S23-S49 (2002).

7. W. Zhou, Y. Yu, and A. Asundi, "Study on aberration suppressing methods in digital micro-holography," Opt. Lasers Eng. 47, 264-270 (2009). 
8. T. Colomb, J. Kühn, F. Charrière, and C. Depeursinge, "Total aberrations compensation in digital holographic microscopy," Opt. Express 14, 4300-4306 (2006).

9. T. Colomb, F. Montfort, J. Kühn, N. Aspert, E. Cuche, A. Marian, F. Charrière, S. Bourquin, P. Marquet, and C. Depeursinge, "Numerical parametric lens for shifting, magnification, and complete aberration compensation in digital holographic microscopy," J. Opt. Soc. Am. A 23, 3177-3190 (2006),

10. L. Miccio, D. Alfieri, S. Grilli, P. Ferraro, A. Finizio, L. De Petrocellis, and S. D. Nicola, "Direct full compensation of the aberrations in quantitative phase microscopy of thin objects by a single digital hologram," Appl. Phys. Lett. 90, 041104 (2007)

11. T. Colomb, E. Cuche, F. Charrière, J. Kühn, N. Aspert, F. Montfort, P. Marquet, and C. Depeursinge, "Automatic proce- dure for aberration compensation in digital holographic microscopy and applications to specimen shape compensation," Appl. Opt. 45, 851-863 (2006).

12. U. Schnars, and W. Jueptner, Digital Holography (Springer, 2005).

13. J. W. Goodman, Introduction to Fourier Optics (McGraw-Hill, 1996).

14. H., Gross, ed., Handbook of Optical Systems: Aberration Theory and Correction of Optical Systems (Wiley-VCH, 2007), Chap. Aberrations.

15. W., Osten, ed., Optical Inspection of Microsystems (Taylor and Francis, 2006).

16. D. Claus, "Resolution improvement methods applied to digital holography," Ph.D. thesis (University of Warwick, 2010) 